\title{
An Analysis of Energy Optimization algorithm in Wireless Sensor Network
}

\author{
Indu Singh \\ (Computer Science and Engineering, India)
}

\begin{abstract}
Wireless sensor networks consist of small nodes with sensing, computation, and wireless communications capabilities. Many routing, power management, and data dissemination protocols have been specifically designed for WSNs where energy awareness is an essential design issue. Routing protocols in WSNs might differ depending on the application and network architecture. In this article we present a survey of stateof-the-art routing techniques in WSNs. We first outline the design challenges for routing protocols in WSNs followed by a comprehensive survey of routing techniques. Overall, the routing techniques are classified into three categories based on the underlying network structure: flit, hierarchical, and location-based routing. Furthermore, these protocols can be classified into multipath-based, query-based, negotiation-based, QoSbased, and coherent-based depending on the protocol operation. We study the design trade-offs between energy and communication overhead savings in every routing paradigm. We also highlight the advantages and performance issues of each routing technique. The article concludes with possible future research areas.

Keyword: Quality of service, QOS-based protocol, Coherent based protocol, Routing protocol and Wireless Sensor Network

\section{INTRODUCTION}

Wireless sensor networks (WSN) represent a new paradigm shift in Adhoc networks. Wireless Sensor Networks consist of small nodes with sensing, computation and communication capabilities. The sensor networks task is to sense the environment, and relay the information back to a remote base station, where the user can access it. Sensor nodes are deployed in a monitoring region to gather information on environments like temperature, humidity, position, sound etc. Many routing protocols have been specifically designed for Wireless Sensor Networks where energy awareness is an essential design issue. Overall, the routing techniques are classified into three categories based on the underlying network structure: flat, hierarchical and location based routing. Energy Conservation is the major issue in distributed wireless sensor network. Routing is a challenging task in WSNs because of their unique characteristics which makes it different from other wired and wireless networks like cellular or mobile ad hoc network (MANETs).
\end{abstract}

\section{ROUTING STRATEgIES IN WSN}

A number of routing protocols have been developed for the WSN till today. Due to its constraints in the processing power and limited battery power, the routing protocols for the wired networks cannot be used here. All the proposed protocols will fall under any of the three categories:

1) Direct approach

2) Location based routing

3) Attribute based routing.

The simple flooding type routing protocols will be coming under the direct approach. Though it is simple in its implementation, it is not an energy efficient protocol for the sensor networks. In the Location based routing the base station communicates with sensor nodes based on its location identity. Here all the nodes are aware of its location through GPS (Global Positioning System) receivers in the network. In WSN instead of collecting information from all the nodes the application needs the data only from the nodes which satisfies its interest and this information gathering technique is widely called as the data centric approach or attribute based routing. Direct diffusion and rumor routing are the best examples for the attribute based routing or data centric approach

\section{Challenges in Wireless Sensor Network:}

Wireless sensor networks are more flexible in obtaining the data from environment. The sensor nodes in conventional wired sensor networks are expensive and demand large amount of energy for operation. Here, summaries some constraints that may alter the design of wireless sensor networks.

Limited energy supply: In wireless sensor network, energy is a main constraint. The operations of a sensor node, such as data processing and transmission are energy-consuming; it is easy to drain the energy of the node during network operation. This problem becomes worsened by the fact that nodes in some applications of 
wireless sensor networks are left unattended. For example, in a field surveillance application, sensor nodes are distributed in an inaccessible territory. Recharging or replacing the node batteries is impossible. Furthermore, the replacement of all node batteries in a large area is costlier and unrealistic. Therefore, limited energy of the node is a crucial challenge for the design of wireless sensor networks.

$\square$ Limited transmission range: The constraint of antenna capability and node energy limits the transmission energy of node. Although some nodes are able to vary their power level so as to adjust the transmission range in specific applications. The maximum transmission area of a sensor node is relatively small as compared to the traditional wireless network. Therefore, in many applications, a large no. of nodes has to be deployed in order to guarantee good network coverage

Small storage size: The storage size of a sensor node is very small as compared with to those in traditional networks. This constraint of sensor node makes the wireless sensor networks unsuitable to be employed in applications that require high data storage capacity. Further, the data processing and data communication becomes limited due to small storage size.

Other challenges, such as quality of data and time latency, do exist in the design of routing protocols for wireless sensor networks. Tradeoffs can be made among these challenges according to the specific requirement of applications.

\section{Objective of the Study}

The main objective of this paper is that I have analyzed energy efficient routing protocol and developed a centralized clustering, energy-efficient routing protocol for wireless sensor networks. The clustering-based structure is used in our protocol to efficiently organize various nodes and utilize limited resource of system (such as energy). The centralized cluster head selection algorithm of our routing protocol is performed by the base station in order to choose better cluster heads by using the global information on the network. These selected cluster heads ensure the intra-cluster data transmission and data transmission from cluster heads to the base station is energy-efficient. Furthermore, the energy dissipation in selected cluster heads can be balanced while using the cluster head selection algorithm of our protocol.

The cluster formation in our protocol is able to balance the load of clusters. Thus, the cluster heads will not drain their energy quickly due to the over-burdened load. The multi-hop routing algorithm of our protocol can reduce the energy consumption related to the data transmission from cluster heads to the base station. Benefitting from both, the energy-efficient data transmission and balanced energy dissipation of nodes, the network lifetime becomes prolonged when using our routing protocol. Since the residual energy of node is considered when choosing cluster heads and intermediate nodes, our protocol can efficiently utilize high-energy nodes in the network. Although our protocol is specific for the wireless sensor network applications, some algorithms, such as the centralized cluster head selection algorithm, can be adopted in the protocols for other wireless networks. In addition to this, the multi-hop routing algorithm used in our protocol can be applied to other wireless network routing (like the ad hoc network routing).

\section{Routing Protocols for Wireless Sensor Networks}

The characteristics of a wireless sensor network that distinguishes it from those in traditional network and wireless ad hoc networks have posed many challenges on the design of routing protocols for wireless sensor networks. First of all, almost all the applications of wireless sensor networks require flow of sensed data from multiple sources (sensor nodes) to a particular sink (the base station) [2, 3]. This many-to-one model for transmission is different from the one-to-one or any-to-any models implemented in conventional wireless conventional networks and wireless ad hoc networks. Secondly, since many sensor nodes may generate the same value within the vicinity of the phenomenon, significant redundancy exists in the data traffic from sensor nodes to the base station [4]. Therefore, the data aggregation technique is strongly desired to be exploited in routing protocols for wireless sensor networks. Thirdly, constraint of sensor nodes in terms of transmission power, onboard energy, processing capacity and storage is rigorous. Especially, the energy supply of a sensor node is limited. Moreover, it is impossible to recharge or replace batteries for numerous sensor nodes that are distributed in remote region in various applications of wireless sensor networks. Therefore, energy efficiency is an important consideration for the design of routing protocol for wireless sensor networks.

Because of these different characteristics, routing protocols for traditional networks and ad hoc networks are no longer suitable for wireless sensor networks. To solve the problem of routing data, many routing protocols have been proposed for wireless sensor networks. The underlying structure can play a significant role in the operation of the routing protocol for wireless sensor networks. According to the structure, these protocols can be classified 
into three main categories: hierarchical-based routing protocol, location-based routing protocol \& flat based routing protocol.

\subsection{Flat-based Routing Protocol}

In flat networks, each node typically plays the same kind of role, and sensor nodes collaborate to perform the sensing task. In many applications of wireless sensor networks, large number of nodes is deployed. Thus, it is not feasible to assign a global identifier to each node in the network. The lack of global identification (such as address) along with random deployment of sensor nodes makes it difficult to select a set of specific nodes to be queried. This consideration has led to data-centric routing, where the sink (base station) sends a query to certain regions and waits for data from the sensor located in the selected regions.

\subsection{Hierarchical-based Routing Protocol}

In order to cope with a large number of sensor nodes in wireless sensor networks, network clustering is implemented in many routing protocols. In a hierarchical or clustering architecture, higher order energy nodes are used to process and transmit the information while low energy nodes are used to perform the sensing task. Furthermore, taking advantage of the clustering mechanism, significant amount of energy can be saved by performing data aggregation that decreases the actual size of the data sent to the base station. Therefore, hierarchical architecture is utilized in routing protocols can contribute for overall system scalability, lifetime, and energy efficiency. Low energy adaptive clustering hierarchy (LEACH) protocol proposed in [2] and its improved protocols, such as LEACH-C, are typical hierarchical-based routing protocols for wireless sensor networks.

2.3 Location-based Routing Protocol In location-based routing protocols for wireless sensor networks, we require information of location for sensor nodes. In this kind of routing protocol, sensor nodes are addressed by means of location. The distance between the two neighboring nodes can be determined on the basis of incoming signal strength. Relative coordinates of nodes can be obtained on exchanging information between neighbors. Alternatively, the location of nodes may be available by direct communication with a satellite, using GPS (global positioning system), if nodes are equipped with a small low-power GPS receiver location-based routing protocols, location information can be utilized in routing data in an energy efficient way. For instance if the region to be sensed is known using the location of sensors, the query can be diffused only to that particular region. Thus, significant energy can be saved by reducing the number of transmissions. Geographic adaptive fidelity (GAF) and Geographic and energy aware routing (GEAR) are two energy-aware location-based routing protocols.

\subsection{Energy Efficiency in Routing}

\section{Energy-Efficient Routing Protocols}

Since energy efficiency is more important for wireless sensor networks than any other networks, more research works have already been done in routing in WSN. In general, data Transmission in wireless communication takes more power than data processing. Whenever the nodes are transmitting more number of data proportionately their battery power also get Reduce. To reduce the data size we can go for data fusion or aggregation techniques. Data fusion is that in which the sensed data from different nodes are fused at certain point suitable for the transmission in its reduced size. Even in the data aggregation concept there are two types of aggregation. The first type of data aggregation fuses the data gathered from different sources and sends the final fused data in reduced size. But the problem behind this approach is it lacks in accuracy and precision of data from various sensor nodes. The second approach combines the data from different sources under the single header and forwards it to the base Station.Here header packets consolidate and pass it to the base station without any modification to the original data from the sensors. Hence accuracy is improved. Study on energy efficient routing in WSN brings this two broad classification of approaches. They are,

Clustering approach

Tree based approach

\section{Clustering techniques:}

Dividing the sensor networks into small manageable units is called as clustering. Though the main reason behind the implementation of the clustering scheme is to improve the scalability of the network, it is an important factor in achieving energy efficient routing of data within the network. Apart from achieving scalability of the network it has more advantages like conserving communication bandwidth within the clusters, avoiding redundant message transfer between the sensor nodes, localizing energy efficient route setup within the clusters. Some of the energy efficient routing protocols based on clustering are LEACH, HEED, DECA, etc 


\section{Tree Based Approach:}

Apart from clustering techniques in WSN, another energy efficient way of routing the data over the network is tree based approach. In this approach a hierarchical manner of aggregation points are formed which resembles the tree structure. The leaves are the source nodes and the root is the sink node. The data when travelling gets aggregated in the intermediate nodes itself. The most successful energy efficient routing protocol which follows the tree based approach was PEGASIS.

Study on energy efficient routing in WSN brings this two broad classification of approaches, clustering and tree based approach. I have studied the energy efficient routing protocol. It consists of a cluster head selection algorithm, a method for cluster formation and inter cluster routing algorithm.

In this thesis, proposed algorithm is to compare with LEACH routing algorithm. In proposed algorithm, the secondary cluster-head can be selected to minimize the searching over-head and with limited amount of energy more data have to be send. As in the LEACH routing algorithm, randomized cluster-head can be selected and it consumes more energy to send data to base station as compared to the proposed routing algorithm.

\subsection{Problem Statement}

The number of nodes deployed in the network area is large. Thus, the overall data flow in the network is considerable and large data flow will incur significant energy dissipation for nodes. In addition to this, the densely deployed nodes incur highly co-related data. Since the nodes are energy constrained, the routing protocol is should be energy-efficient. In addition, because the energy consumption is different from node to node due to various functions and positions in the network, the protocol should be able to balance the energy dissipation of nodes. The distances of nodes to the base station are usually long in a wireless sensor network. Long distant data transmission will count for considerable energy dissipation. Thus, the routing protocol should be able to consume less energy. Therefore, the problems that need to be addressed in the design of routing protocol for wireless sensor networks can be summarized as:

How to efficiently organize numerous nodes in the network in order to reduce the energy dissipation of nodes.

How to Balance the energy consumption of nodes

$\checkmark$ How to minimize the energy dissipation of data transmission from sensor nodes to the base station

\subsection{Proposed Solution}

In proposed approach, sensor nodes form a cluster and in order to send the data to base station clusterhead is selected based on the energy and that sensor nodes is Selected as a $\mathrm{CH}$ (cluster-head). While processing of Cluster-head node the energy become reduce, so if the energy of $\mathrm{CH}$ is becomes below the non-cluster-head nodes energies means next round should to be processed. In the next round, the Secondary cluster-head should be made as a lead while selection of cluster-head for the first round, so no need to select the cluster-head for next round. In this case, searching overhead to be minimized. As important that, this protocol approach consumes limited energy to send the data.

\section{LEACH}

\section{Proposed Method \& LEACH Approach}

The main Aim of the LEACH routing protocol is to efficiently maintain the Energy consumption and performing data aggregation in order to reduce the number transmitted messages to the sink. The operation of LEACH[3] is divided in two phases,

(i) Set-up phase.

(ii) Steady state phase.

\section{Set-up phase}

In set-up phase, the cluster head is selected and then it forms a group . So after some time the corresponding cluster head energy to be reduced and to rotate the cluster head selection process.

\section{Steady State phase}

In steady state phase, the groups are formed and the corresponding cluster head is selected. After the cluster head receives the data, it can be aggregated and the data can be transmitted to the base station [

Eligibility for cluster-head

In order to select the cluster-head, it will satisfy the eligibility criteria. First, average energy to be calculated and then sensor nodes which exceeds energy level than the average energy considered as eligible for Selection of cluster-head.

\subsection{Pseudo code \\ 4.1.1 CLUSTER-HEAD SELECTION PROCESS \\ During the selection of cluster-head \\ if}


Sensor nodes $>$ than average energy level

then

Sensor nodes eligible for cluster-head

Else

Not eligible for Cluster-head selection process

Start the process.

\subsubsection{LEACH APPROACH}

Randomized Selection of Cluster-head $(\mathrm{CH})$

If

Random Cluster-head acts up to when $\mathrm{CH}<$ than non $\mathrm{CH}$ nodes

Then

Next randomized node selected as $\mathrm{CH}$ for another round and the $\mathrm{CH}$

For first round is stored in dummy variable to 50 avoid re-Cluster head selection process.

Else

Already selected $\mathrm{CH}$ to be re-used, for that performance to be reduced

Else if

Not eligible for $\mathrm{CH}$ selection process.

\subsubsection{Proposed Approach}

Secondary $\mathrm{CH}$ to be selected as a Back-end node with the selection of $\mathrm{CH}$ for First round

If

For Selection of Secondary $\mathrm{CH}$, the nodes which have more energy level after the selection of $\mathrm{CH}$ for first round acts as a backend node.

Then

After the reduction in energy level for first round as compare to Average energy without selecting the $\mathrm{CH}$ for next round the Secondary $\mathrm{CH}$ as a Back-end acts and it can be act for next round.

Else

Reselection of $\mathrm{CH}$ leads to be a selection Over-head.

Else if More Energy Consumption.

\subsection{Cluster Head selection Algorithm}

In the cluster-based protocol, the nodes are organized into local clusters. Each cluster consists of one cluster head and no. of member nodes (the non-cluster-head nodes belong to the same cluster). All non-clusterhead nodes must transmit their data to the cluster head, while the cluster head must receive data from all the cluster members, perform aggregation on the data and transmit processed data to the remote base station. Therefore, being a cluster head is much more energy-intensive than being a non-cluster head node. In the scenario where all nodes are energy-limited, it is necessary to choose appropriate cluster heads for the protocol.

\subsubsection{Weight-Based Cluster Head Selection}

In the proposed protocol, a centralized weight-based cluster head selection algorithm is proposed. This algorithm aims at choosing cluster heads that ensure both intra cluster data transmission and inter cluster data transmission are energy efficient. In addition, the energy balance is considered when designing this algorithm. This algorithm is performed by the base station which has no energy constraint.

Cluster heads are the local centers in their own clusters. They perform many energy-consuming tasks such as collecting data from member nodes and forwarding processed data to the base station. Thus, the no of neighboring nodes, the distances between cluster head and member nodes, and the distances between cluster heads and the base station are all crucial to choose cluster heads. In addition to this, to choose nodes with to be the cluster heads with more energy, residual energy of nodes is considered for cluster head selection in the protocol.

We define the set of nodes in the network as $\mathrm{V}=\{\mathrm{v} 1, \mathrm{v} 2, \ldots \ldots \mathrm{vn}\}$, where $\mathrm{N}$ is the number of nodes in the network. The cluster head selection algorithm is performed as following:

Step 1: Find the neighbors of each node v (i.e, nodes within the transmission range of node $\mathrm{v}$ ) as:

$\mathrm{N}(\mathrm{v})=$ 
Where $\mathrm{N}(\mathrm{v})$ is the number of the neighbors of node $\mathrm{v}$, dist $(\mathrm{v}, \mathrm{u})$ is the distance between node $\mathrm{v}$ and node $\mathrm{u}$, and $\mathrm{R}$ is the intra-cluster transmission range.

Step 2: Compute the degree-difference for every node v:

$\Delta \mathrm{v}=\mid \mathrm{N}(\mathrm{v})-\delta\}$,

Where $\Delta \mathrm{v}$ is the degree-difference of node $\mathrm{v}$ and $\delta$ in the ideal number of nodes in a cluster.

Step 3: Calculate the sum of square distance between node $v$ and its neighbors as

$\mathrm{d}=(\mathrm{v}, \mathrm{u}) 2\}$,

where $\mathrm{d}$ is the sum of square distance between node $\mathrm{v}$ and its neighbors .

Step 4: Compute the Dv for each node $v$

$\mathrm{Dv}=\mathrm{D}(\mathrm{v}, \mathrm{BS})$,

Step 5: Estimate the residual energy of every node v, Ev .

Step 6: Calculate the combined weight of each node $v$ from :

$\mathrm{Wv}=(\mathrm{w} 1 \Delta \mathrm{n}+\mathrm{w} 2 \mathrm{~d}+\mathrm{w} 3 \mathrm{Dv})$ Eini/Ev,

Where $\mathrm{Wv}$ is the combined weight of node $\mathrm{v}, \mathrm{w} 1, \mathrm{w} 2$ and $\mathrm{w} 3$ are the neighboring parameters that determined by the system property and protocol requirements, and Eini is the initial energy of the node .

Step 7: Select the node with the smallest Wv as the cluster head. All the neighbors of the selected cluster head are no longer allow participating in the remaining part of selection procedure.

Step 8: Repeat steps 2-7 for the following nodes until all the nodes have been selected as a cluster head or assigned to a cluster.

\subsubsection{Cluster Formation}

The cluster formation of the centralized protocol aims at balancing the energy load of cluster heads. Once the nodes have been selected as a cluster heads, they will invite other non-cluster head nodes in the network to join the clusters. Each cluster head broadcasts an invitation message using a non-persistent carriersense multiple access (CSMA) MAC protocol. The cluster head selection algorithm used in our protocol ensures that the selected cluster heads can cover all the nodes in the network with limited transmission range.

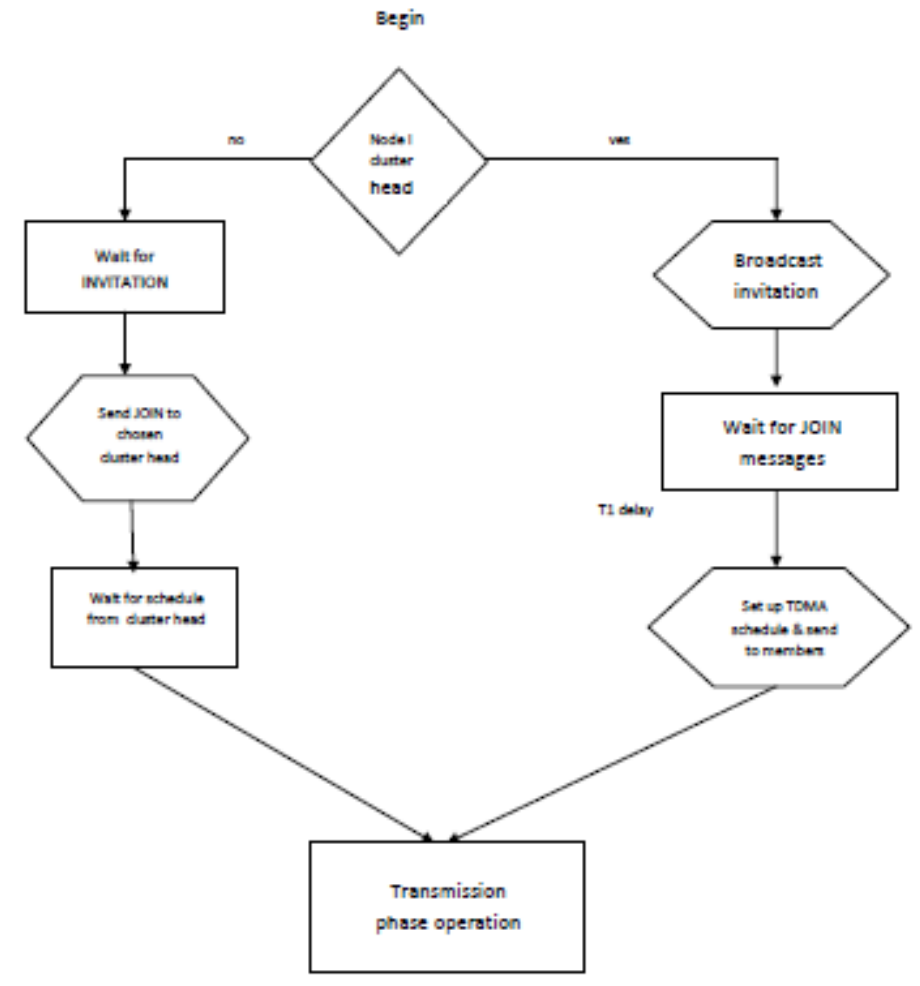

\section{Figure flow of cluster formation}

In order to reduce the interference of transmission, all cluster heads load a random time delay $\mathrm{t} 1$, after which they broadcast the invitation messages. The random time delay $\mathrm{t} 1$ should be set appropriately to ensure there is enough interval time between broadcasting an invitation by two random cluster heads without incurring considerable time delay of the system. 55 
Since some ordinary nodes may reside in multiple neighborhoods of cluster heads, they will receive more than one invitation messages (if a cluster head receives invitation messages from other cluster heads, it will just discard the messages).

\section{CONCLUSION}

I have analyze a centralized clustering, energy-efficient routing protocol that meets the energy challenges in wireless sensor networks. A centralized cluster head selection algorithm which is performed by the base station is utilized in the protocol to choose cluster heads using global information of the network. Using this centralized algorithm, better deterministic cluster heads in terms of energy-efficiency and energy distribution can be selected. The cluster formation scheme in the protocol aims at distributing energy load among cluster heads. This can avoid the situation that some cluster heads may die earlier than others due to the heavy load. In order to reduce the energy consumption for data transmission from cluster heads to the base station, an energy-efficient routing algorithm is developed in the protocol. This algorithm conducts a multi-hop routing based on an optimal transmission range in terms of energy-efficiency. Significant energy dissipation of data transmission can be reduced by using this routing algorithm.

\section{References}

[1] K. Khamforoosh, and H. Khamforoush, -A new rounting Algorithm for Energy Reduction in Wireless Sensor Networksl, IEEE, 2010

[2] C. Prehofer and C. Bettstetter, "Self-organization in communication networks: principles and design paradigms," IEEE Communications Magazine, vol. 43, pp. 78-85, 2009

[3] Y. Yang, H.-H. Wu, H.-H. Chen, Short: Shortest hop routing tree for wireless sensor networks, in: Proceedings of IEEE ICC-2009, 2009.

[4] I. F. Akyildiz et al., -Wireless Sensor Networks: A Survey,\|l Elsevier Sci. B. V. Comp. Networks, vol. 38, no. 4, Mar. 2009, pp. $393-422$.

[5] Ming Liu, Jiannong Cao, Guihai Chen, and Xiaomin Wang, -An Energy-Aware Routing Protocol in Wireless Sensor Networksl, Sensors 2009, vol. 9, pp. 445-462.

[6] W. R. Heinzelman, A. P. Chandrakasan, and H. Balakrishnan, -Energy- Efficient Communication Protocol for Wireless Microsensor Networks, $\|$ Proc. 33rd Hawaii Int‘l. Conf. Sys. Sci., Jan. 2007.

[7] Jamal N. Al-Karaki, Ahmed E. Kamal, \ Routing Techniques in Wireless Sensor Net- works: A Survey," IEEE Wireless Communications, Vol 11. pp.6-28, Dec. 2006.

[8] W. Heinzelman, A. Chandrakasan, and H. Balakrishnan, \An application- specific protocol architecture for wireless microsensor networks," IEEE Transaction on Wireless Networking, 2006.

[9] C. F. Garca-Hernndez, P. H. Ibargengoytia-Gonzlez, J. Garca-Hernndez, and J. A. Prez-Daz, "Wireless sensor networks and applications: a survey, International Journal of Computer Science and Network Security, vol. 7, no. 3, pp. 264-273, March 2006.

[10] Y. He, Y. Ji, Y. Zhang, and X. Shen, "A new energy efficient approach by separating data collection and data report in wireless sensor networks," in IWCMC'06, Vancouver, July 2006, pp. 1165-1170. 66

[11] M. J. Handy, M. Haase, and D. Timmermann, "Low energy adaptive clustering hierarchy with deterministic cluster-head selection," in Forth IEEE Conference on Mobile and Wireless Communications Networks, Stockholm, September 2006, pp. 368-372.

[12] H. Lou, J. Luo, Y. Liu, and S. K. Das, "Energy efficient routing with adaptive data fusion in sensor networks," in DIALMPOMC'05, Cologne, September 2005, pp. 80-88.

[13] V. Mhatre and C. Rosenberg, "Design guidelines for wireless sensor networks: Communication, clustering and aggregation," Ad Hoc Networks, vol. 2, no. 1, pp. 45-63, 2004

[14] T. Huynh and C. Hong, "An energy delay efficient multi-hop routing scheme for wireless sensor networks," IEICE, vol. E89-D, no. 5, pp. 1654-1661, May 2004.

[15] Y. Xu, J. Heidemann, and D. Estrin, "Geography-informed energy conservation for ad hoc routing," in Seventh Annual ACM/IEEE International Conference on Mobil Computing and Networking, Rome, July 2002, pp. 70-84. 